\title{
Rhabdomyolysis with acute anuria renal failure caused by surgical injection-induced tetanus in an adult
}

\author{
Authors: Lei Li ${ }^{\mathrm{A}}$ and Zhenning Liu ${ }^{\mathrm{B}}$
}

Tetanus is a rapidly progressive infection characterised by painful spasms and muscular rigidity with a series of complications including airway obstruction, respiratory failure, pulmonary infection, heart arrhythmias and acute kidney injury. Rhabdomyolysis with acute anuria renal failure is rarely reported as a complication in tetanus. We present a case of an adult who suffered from rhabdomyolysis combined with acute anuria renal failure caused by surgical injection-induced tetanus. This patient was treated with tetanus immunoglobulin, control of muscle spasm, mechanical ventilation and haemoperfusion plus continuous veno-venous haemofiltration (CVVH). The patient fully recovered and was discharged from hospital 5 weeks after admission. Good bowel preparation is an important prerequisite for preventing tetanus in a haemorrhoids operation. Effective control of muscle spasms with neuromuscular blockers and early administration of haemoperfusion plus CVVH are vital to the treatment of tetanus-induced rhabdomyolysis with acute renal failure.

KEYWORDS: tetanus, rhabdomyolysis, acute renal failure

DOI: $10.7861 /$ clinmed.2020-0841

\section{Introduction}

Although it is rare in developed countries, tetanus is a serious and life-threatening public health disease in developing countries. About 60,000 people worldwide die from tetanus every year. Tetanus is a rapidly progressive illness characterised by painful spasms and muscular rigidity. The clinical manifestations are due to a potent neurotoxin produced by Clostridium tetani. The common complications of tetanus include cardiovascular system instability, airway obstruction, pulmonary infection and respiratory failure. ${ }^{1}$ Rhabdomyolysis combined with acute anuria renal failure is a rare complication of tetanus in adults. Here, we describe a severe case of tetanus complicated with rhabdomyolysis and acute renal failure in an adult after surgical injection in haemorrhoids. Luckily, he was treated successfully

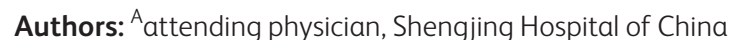
Medical University, Shenyang, China; ${ }^{\text {B }}$ rofessor of medicine, Shengjing Hospital of China Medical University, Shenyang, China with tetanus immunoglobulin, neuromuscular blockers and blood purification.

\section{Case presentation}

A 37-year-old, previously healthy, male presented to the emergency department with progressive limitation in mouth opening and painful muscle spasms in his right thigh for 3 days. Eleven days prior, he was treated with automatic ligation of haemorrhoids in combination with haemorrhoid injection at a local clinic. He denied fevers, headache or other postoperative discomfort after surgery. On physical examination, he was alert, slightly febrile $\left(37.6^{\circ} \mathrm{C}\right)$ and normotensive, with oxygen saturation $97 \%$ on room air. He had intermittent sinus tachycardia accompanied with muscle cramps every 10-20 minutes. His abdomen was non-tender and there was slight exudation in the surgical site. Computed tomography demonstrated neither perianal abscess nor pelvic infection (supplementary material S1; Fig S1a and S1b), except the slight infection of the lower lobe of both lungs (supplementary material S1; Fig S1c and S1d). Laboratory investigations revealed an increased white blood cell count $\left(12.2 \times 10^{9} / \mathrm{L}\right)$ with C-reactive protein (CRP; $46.2 \mathrm{mg} / \mathrm{L})$, and elevated myoglobin $(2,181 \mu \mathrm{g} / \mathrm{L})$ and creatine kinase $(10,243 \mathrm{U} / \mathrm{L})$. The other laboratory tests including haemoglobin, platelet count, liver enzymes, bilirubin and creatinine were normal.

\section{Diagnosis}

He was suspected to have tetanus complicated with rhabdomyolysis and pneumonia.

\section{Initial management}

Immediately, horse tetanus immunoglobulin was injected intramuscularly, and then metronidazole and penicillin were intravenously infused every 6 hours. The muscle spasms were controlled by diazepam, midazolam or propofol, depending on the severity of the spasms. Simultaneously, in addition to liquid supplement, sodium bicarbonate was administered to alkalise urine in order to excrete the myoglobin. The surgical site was locally disinfected with hydrogen peroxide and povidone-iodine.

\section{Case progression and outcome}

Although he was treated aggressively after admission, the symptoms of muscle cramps were only partially improved. On day 3 
Fig 1. Laboratory markers and major clinical course. AST = aspartate aminotransferase.

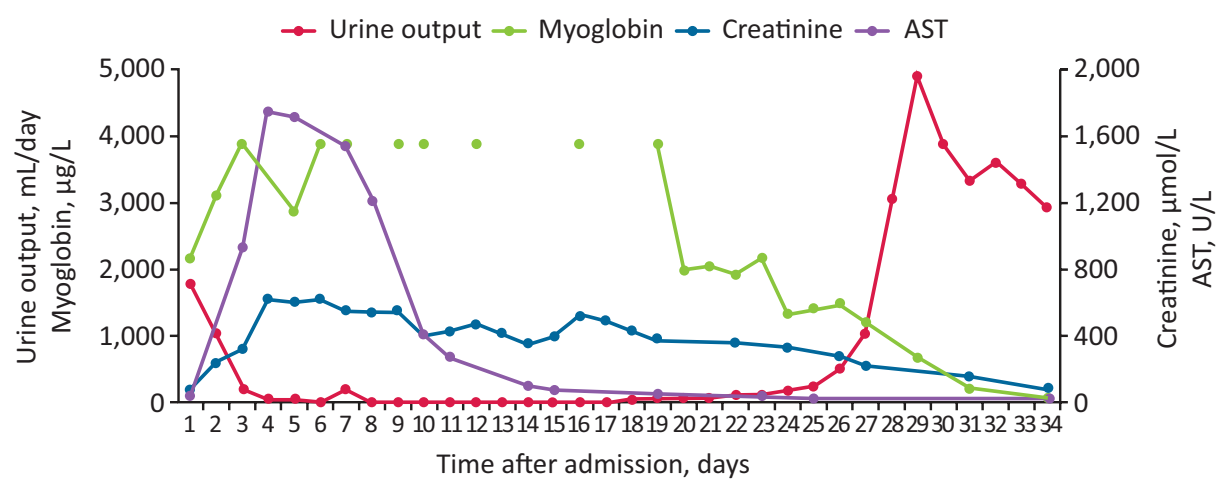

of admission, he was mechanically ventilated after emergency tracheotomy due to dyspnoea caused by phlegm obstruction in the airway. On day 4 of admission, the high levels of serum creatine kinase $(20,105 \mathrm{U} / \mathrm{L})$ and myoglobin $(>3,890 \mu \mathrm{g} / \mathrm{L})$ indicated that the rhabdomyolysis was not effectively treated. To further attenuate the muscle cramps, atracurium besylate was administrated. In addition, the poor urine output ( $210 \mathrm{~mL} /$ day) accompanied with the increased serum creatinine $(327.1 \mu \mathrm{mol} / \mathrm{L})$ and urea (22.77 mmol/L) indicated acute kidney injury. Haemoperfusion was performed immediately with HA330 disposable haemoperfusion cartridge to remove excess myoglobin from blood. Subsequently, continuous renal replacement therapy (CRRT) was initiated for the management of rhabdomyolysis-induced acute kidney injury. The daily duration of haemoperfusion and CRRT were 2 hours and 12-16 hours, respectively. After 4 weeks of admission to the intensive care unit, CRRT was terminated resulting from the recovered renal function as indicated by the increased urine output (1,050 mL/day) accompanied with the reduced serum creatinine $(219.3 \mu \mathrm{mol} / \mathrm{L})$ and urea $(11.48 \mathrm{mmol} / \mathrm{L})$. Considering the improvement of respiratory function, he was weaned off the ventilator on day 30 . He had fully recovered with normal mouth opening and renal function, and consequently discharged from hospital 5 weeks later. The laboratory markers and major clinical course of the patient are shown in Fig 1. At 1-year follow-up, the patient was free of tetanus symptoms with no sequelae and his follow-up laboratory examination results were normal.

\section{Discussion}

Tetanus is caused by Clostridium tetani, a Gram-positive bacillus, which can be isolated from soils and stools of domestic animals and humans. In addition to contamination of wounds with manure, or rusty metal, tetanus can also occur after snakebites, intramuscular injections and surgery. ${ }^{1}$ Under anaerobic conditions, $C$ tetani can produce two toxins: tetanospasmin (commonly termed tetanus toxin) and tetanolysin during growth. Tetanospasmin leads to the persistent tonic spasms with violent exacerbation via the inhibition of neurotransmitter release. Tetanus usually follows a recognised injury with an incubation period of about 7-10 days. In this case, he developed progressive muscle tension 8 days after surgery. Considering the existence of C tetani in human faeces, contamination of the surgical site was mainly responsible for this accident. Nevertheless, there are no guidelines that tetanus prevention is recommended routinely after anorectal surgery. However, Janicke and Pundt have proposed that tetanus prophylaxis and intravenous antibiotics should be administrated in all but superficial rectal tears. ${ }^{2}$ Further studies should be performed in the future to testify the feasibility and value of tetanus prevention in anorectal surgery.

Vigorous and repeated contractions of muscles during muscle spasms, coupled with hypoxia of these muscles, may therefore result in muscle cell necrosis leading to acute rhabdomyolysis. Initial management including adequate hydration, alkalisation of urine and control of infection is the key to a better outcome of rhabdomyolysis. Additionally, neuromuscular blocking agents may be required for the relief of muscle spasms and exert beneficial effects on the relief of tetanus-induced rhabdomyolysis.

Acute kidney injury induced by tetanus was previously reported as a serious complication, however, (as listed in supplementary material S2; Table S1) most of the cases were nonoliguric, mild and transient. ${ }^{3}$ Rhabdomyolysis due to uncontrolled muscle spasms and autonomic dysfunction characterised by hemodynamic instability and adrenergic overactivity were considered as potential mechanisms for acute renal insufficiency. ${ }^{3}$ Acute kidney injury increases risk of death, prolonged hospitalisation and ventilator days, and increases hospital costs. ${ }^{4}$ Continuous veno-venous haemofiltration (CVVH) can be employed to effectively improve kidney function and remove myoglobin resulting in obstruction and toxicity to the renal tubules. ${ }^{5}$ Also, haemoperfusion is beneficial for the treatment of patients with rhabdomyolysis. ${ }^{6}$

\section{Conclusion}

Good bowel preparation is an important prerequisite for preventing tetanus, since contamination of $C$ tetani in surgical sites was the radical cause of this accident. Effective control of muscle spasms and blood purification should be established early in the treatment of tetanus-induced rhabdomyolysis with acute renal failure.

\section{Key points}

> Rhabdomyolysis with acute anuria renal failure is a rare complication in tetanus.

> Bowel preparation is an important prerequisite for preventing tetanus.

> Effective control of muscle spasms and early blood purification are vital. 


\section{Supplementary material}

Additional supplementary material may be found in the online version of this article at www.rcpjournals.org/clinmedicine: S1 - Computed tomography demonstrating neither perianal abscess nor pelvic infection, except a slight infection of the lower lobe of both lungs.

S2 - Reports of tetanus-induced rhabdomyolysis in non-neonatal cases, 2000-2019.

\section{Funding}

This work was supported by the Key Science Research Plan of Science and Technology Department of Liaoning Province (grant no: 2018225095), and the 345 Talent Project of Shengjing Hospital of China Medical University.

\section{References}

1 Cook TM, Protheroe RT, Handel JM. Tetanus: a review of the literature. $\mathrm{Br}$ ] Anaesth 2001;87:477-87.
2 Janicke DM, Pundt MR. Anorectal disorders. Emerg Med Clin North Am 1996;14:757-88.

3 Daher EF, Abdulkader RC, Motti E et al. Prospective study of tetanus-induced acute renal dysfunction: role of adrenergic overactivity. Am J Trop Med Hyg 1997;57:610-4.

4 Naseem F, Hussain A, Arif F. Frequency of acute kidney injury in tetanus patients of paedriatic intensive care unit: a public hospital experience. Pak J Med Sci 2018;34:363-7.

5 Zhang L, Kang Y, Fu P et al. Myoglobin clearance by continuous venous-venous haemofiltration in rhabdomyolysis with acute kidney injury: a case series. Injury 2012;43:619-23.

6 Nicolau D, Feng YS, Wu AH et al. Myoglobin clearance during continuous veno-venous hemofiltration with or without dialysis. Int J Artif Organs 1998;21:205-9.

Address for correspondence: Dr Zhenning Liu, Department of Emergency Medicine, Shengjing Hospital of China Medical University, 36 Sanhao Street, Heping District, Shenyang City, China.

Email: liuzn999@hotmail.com 